\title{
Effects of ethanol and partial reinforcement upon runway acquisition
}

PHILIP B. NELSON AND KEITH A. WOLLEN

WASHINGTON STATE UNIVERSITY

\begin{abstract}
Abstraet
When injected with a placebo, $50 \%$ reward resulted in faster running speeds at the end of training than $100 \%$. No differences were found between 50 and $100 \%$ reinforcement for Ss given ethanol. Running speeds in both placebo and alcohol groups dropped when the Ss were shifted to the opposite drug. The data were interpreted as support for the Amsel-Spence frustration hypothesis.

\section{Problem}

Several Es have found that partially rewarded Ss run faster at asymptote than do those continuously reinforced (e.g., Goodrich, 1959; Logan, 1960; Wagner, 1961). First Amsel (1958) and then Spence (1960) hypothesized that this superiority was due to an increased level of motivation contributed by "anticipatory frustration." As Barry, Wagner, \& Miller (1962) suggested, depressants such as ethanol and sodium amytal might be expected to reduce anticipatory frustration. This in turn should reduce the asymptotic superiority conincident with partial reinforcement. Wagner (1963) tested this hypothesis using sodium amytal. In the drugged groups, $50 \%$ reinforcement resulted in slower asymptotic running, but the typical superiority was obtained in the placebo groups. To the extent that alcohol and sodium amytal have similar effects upon behavior, as was demonstrated in one study (Barry et al, 1962), ethanol should also reduce anticipatory frustration and consequently reduce or eliminate the asymptotic superiority of partially rewarded Ss. This hypothesis was tested (1) by comparing partial and continuous groups trained with ethanol with corresponding control groups trained using a placebo; and (2) by shifting the control groups to ethanol after the partial Ss attained asymptotic superiority. ${ }^{1}$
\end{abstract}

\section{Method}

The apparatus consisted of a 12-in start box, a 36 -in runway, and a 12-in goal box. All sections were 3.5 in wide by 4.7 in high (inside dimensions), painted flat black, and covered with transparent Plexiglas. The start box was separated from the runway by two guillotine doors, one opaque (black) and the other transparent. The opaque door was adjacent to the start box. A similar opaque, retrace door separated the goal box from the runway. The Ss were 36 naive, male, hooded rats which ranged from 98 to 114 days of age on the first day of acquisition.

As preliminary training, the Ss were placed on a 23$\mathrm{hr}$. food and water deprivation schedule which permitted $1 \mathrm{hr}$. of ad lib feeding per day. This schedule was maintained throughout the experiment. On the ninth through fourteenth day of deprivation, each $\mathrm{S}$ was handled for $1 \mathrm{~min}$. , and given 10 Noyes $45 \mathrm{mg}$ reward pellets prior to each day's feeding. Also on these days, the Ss were adapted to a Baxter K-31 infant feeding tube by inserting the tube into the esophagus once daily.

For the acquisition phase, the Ss were divided into four groups identified on the basis of whether they received ethyl alcohol (A) or a dextrose (D) solution, and whether they received 50 or $100 \%$ reinforcement. Thus Group A-50 received alcohol and was reinforced on 50\% of the trials, Group D-100 received dextrose and was reinforced $100 \%$ of the time, etc. The solutions used were $15 \%$ concentration (vol/vol) of ethanol in water, and a caloric equivalent dextrose solution. In both cases, the amount of solution injected was $1.5 \mathrm{cc}$ per $100 \mathrm{gm}$ of body weight at the time of injection.

Each day of acquisition began with the appropriate injection accomplished by stomach loading using a syringe connected to the end of the feeding tube. The Ss were injected approximately $1.5 \mathrm{hr}$. prior to being fed. Twenty min. following injection, a trial was started by placing $S$ in the start box. The opaque start door was opened as soon as S oriented toward it. Exactly $1 \mathrm{sec}$. later, the transparent door was raised automatically by means of a motor. When $\mathrm{S}$ intercepted a photobeam 6 in down the alley, a clock started. The interception of a second beam 6 in further down the alley stopped the clock. Running times over this segment were recorded to the nearest $.01 \mathrm{sec}$. and converted to speed scores in $\mathrm{ft} / \mathrm{sec}$. When $\mathrm{S}$ entered the goal box, the retrace door was lowered. All Ss were given six $45 \mathrm{mg}$ Noyes pellets on each rewarded trial. The $S$ was removed from the goal box after eating the pellets, or after $10 \mathrm{sec}$. on non-rewarded trials. Then $\mathrm{S}$ was placed in a waiting cage for an intertrial interval of $70 \mathrm{sec}$. following which another trial was given.

Acquisition trials began on the fifteenth day of deprivation and continued for a total of 19 days. One trial was given on the first day, three on the second, and four on each subsequent day. The sequence of rewarded and non-rewarded trials for the 50\% groups was obtained by repeating this sequence: +-+--+-++--+-+ +- . On the last two days of acquisition, both of the alchohol groups were given dextrose and both of the dextrose groups received alcohol. The experiment was run in four replications of $12 \mathrm{Ss}$ each.

\section{Results and Discussion}

The daily mean running speeds are presented in Fig. 1. When the mean running speeds over Days 13 through 17 


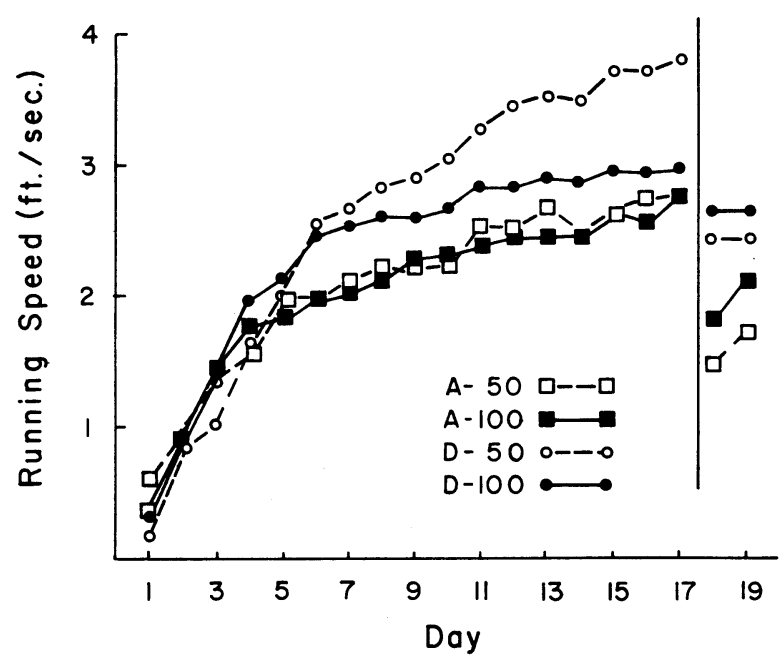

Fig. 1. Mean running speeds as a function of drug condition and percentage reinforcement. The post-shift data are presented to the right of the vertical line.

were examined, the difference between 50 and $100 \%$ was significant for the dextrose groups $(t=4.07, \mathrm{df}=16$, $p<.001)$, but not for the alcohol groups $(t=.98)$. The superiority of the partially reinforced dextrose Ss is consistent with the data from previous studies (e.g., Goodrich, 1959; Logan, 1960; Wagner, 1961) and with the interpretation that the superiority is due to anticipatory frustration. The lack of a difference between the alcohol groups is in agreement with the hypothesis that alcohol reduced frustration and thereby eliminated the difference between 50 and $100 \%$ reward.

The hypothesis that alcohol reduces anticipatory frustration is also confirmed by the drug reversal data. As predicted, the difference between Groups D-50 and D-100 was eliminated after the shift to alcohol. Moreover, the performance of both groups dropped to the same level as the alcohol groups prior to the shift.

Groups A-50 and A-100 each dropped to a lower level than before the shift, with no statistically significant difference between the groups $(t=1.38, d f=16)$. One might expect that removal of alcohol would result in faster running rather than slower. One possible posthoc interpretation ${ }^{2}$ of the drop is that the response of running became conditioned in part to the cues associated with alcohol. If this were true, the removal of these cues might be expected to result in lower performance because of a generalization decrement.

Another related interpretation ${ }^{2}$ of the post-shift drop in Groups A-50 and A-100 is that alcohol reduced the S's fear of the apparatus. On Day 1 of training, the alcohol Ss ran significantly faster than placebo groups $(t=2.21, d f=34, p<.05)$. A similar finding by Barry et al (1962) was interpreted as evidence that alcohol prevented the occurrence of fear-produced inhibition which was presented in the placebo groups. If this were correct, Groups A-50 and A-100 would experience fear of the apparatus for the first time when run with dextrose. Thus fear of the apparatus could have resulted in inhibition much as it does in the early stages of learning. Little or no such inhibition would be expected in the dextrose groups since they would have had 64 trials without alcohol in which to extinguish their fear of the apparatus.

\section{References}

Amsel, A. The role of frustrative non-reward in noncontinuous reward situations. Psychol. Bull., 1958, 55, 102-119.

Barry, H., III, Wagner, A. R., \& Miller, N. E. Effects of alcohol and amobarbital on performance inhibited by experimental extinction. J. comp. physiol. Psychol., 1962, 55, 464-468.

Goodrich, K. P. Performance in different segments of an instrumental response chain as a function of reinforcement schedule. J. exp. Psychol., 1959, 57, 57-63.

Logan, F. A. Incentive. New Haven: Yale University Press, 1960.

Spence, K. W. Behavior theory and learning. Englewood Cliffs, N. J:: Prentice-Hall, 1960.

Wagner, A. R. Effects of amount and percentage of reinforcement and number of acquisition trials on conditioning and extinction. J. exp. Psychol., 1961, 62, 234-242.

Wagner, A. R. Sodium amytal and partially reinforced runway performance. J. exp. Psychol., 1963, 65, 474-477.

\section{Notes}

1. Appreciation is expressed to J. D. Keehn for suggesting the post-shift phase of this experiment.

2. This interpretation is the responsibility of the second author. 